Apidologie, 1980, 11 (1), 95-103.

\title{
RELATIONS ENTRE LA MORPHOLOGIE, L'IMPLANTATION ET LA FONCTION \\ DES SOIES SCOPALES D'EXOMALOPSIS BILIOTTII TORRE-GROSSA (HYMENOPTERA, ANTHOPHORIDAE)
}

\author{
Beziehungen zwischen der Morphologie, der Implantation \\ und der Funktion der skopalen Borsten von Exomalopsis biliottii \\ Torre-Grossa (Hym., Anthophoridae)
}

\author{
J.-P. TORRE-GROSSA \\ I.N.R.A., Station de Zoologie et de Lutte biologique \\ Domaine "Duclos" \\ F. 97170 Petit-Bourg (Guadeloupe)
}

\begin{abstract}
RÉSUMÉ
La microscopie électronique à balayage a permis la description morphologique des différents types de soies constituant les scopae d'une petite abeille anthophoride, Exomalopsis biliottii, pollinisatrice des Solanacées cultivées à la Guadeloupe. Dans le cadre d'une étude sur le comportement de butinage de cet insecte, les relations entre la morphologie, l'implantation et la fonction des soies scopales ont été analysées. Il apparaît que ses relations sont marquées pour deux types de soies :

- La soie simple, implantée sur la face interne du basitarse et servant à brosser le pollen soit sur le corps de l'insecte soit sur les fleurs.

- La soie plumeuse abondamment représentée sur la face externe des tibia et basitarse où est concentre le pollen en vue de son transport.

Une soie extra-scopale présente un intérêt en raison de sa morphologie adaptée probablement à la protection de l'articulation fémuro-tibiale.
\end{abstract}

\author{
SUMMARY \\ RELATIONS BETWEEN MORPHOLOGY, IMPLANTATION AND FUNCTION \\ OF SCOPAE BRISTLES OF EXOMALOPSIS BILIOTTII \\ TORRE-GROSSA (Hym., Anthophoridae)
}

The scanning microscopy made possible to describe the morphology of the different kinds of bristles that form the scopae of a small anthophorid bee, Exomalopsis biliottii, a pollinator of some Solanaceae 
cultivated in the Guadaloupe. In connection with a study about the foraging behaviour of this bee, the relations between morphology, implantation and function of the scopae bristles were analyzed. These relations exist for two kinds of bristles :

- the simple bristle, which is situated on the inner side of the basitarsus and used for brushing pollen either on the insect body or on flowers,

- the feathered bristle, which is abundant on the outer side of the tibia and the basitarsus, gathers pollen in order to carry it.

A bristle outside the scopa is of some interest because of its morphology which is probably adapted to the protection of the femur-tibia joint.

\section{INTRODUCTION}

Les Solanacées cultivées à la Guadeloupe sont visitées par une petite abeille anthophoride, Exomalopsis biliottii TORRE-GrossA*.

Dans le cadre d'une étude générale sur cet insecte et plus particulièrement sur son comportement de butinage nous avons analysé au microscope électronique à balayage et selon les méthodes décrites dans les travaux de Pasteels et Pasteels (1972, 1973 et 1974), les relations entre la morphologie, l'implantation et la fonction des soies scopales. D'autres soies situées à l'extérieur de la scopa et semblant présenter un intérêt particulier sont également décrites.

\section{MATÉRIELS ET MÉTHODES}

Les pattes postérieures des femelles Exomalopsis sont nettoyées de leur pollen à laaide du jet d'eau très fin d'une pipette Pasteur actionnée par une poire en caoutchouc. Après séchage elles sont fixées sur un porte-objet et métallisées, sous vide, à l'or. Les soies sont examinées au microscope électronique à balayage, modèle JSM 35, en orientant les pattes sous différents angles.

Les scopae sont les parties du corps des abeilles butineuses sur lesquelles sont concentrés et transportés les grains de pollen. Elles se caractérisent par une longue et abondante pilosité et se situent généralement sur les pattes posterieures, plus rarement sur les sternites abdominaux et quelquefois sur les deux (MICHENER, 1974).

Chez les femelles d'Exomalopsis on situe facilement les scopae qui se répartissent entre le tibia et le basitarse des pattes postérieures conférant à celles-ci un aspect renflé et coloré.

\section{RÉSULTATS}

Par souci d'homogénéité et dans la mesure du possible, nous avons employé (Tabl. 1) la terminologie descriptive de Pasteels et Pasteels (1972).

* Nouvelle espèce décrite par l'auteur dans "Systématique et répartition géographique des Exomalopsis dans l'arc antillais ", Rev.fr. Entomol., 1980 (sous presse). 
TABL. 1. - Description, implantation et fonctions probables des différents types de soies.

TABL. 1. - Beschreibung, Implantation und wahrscheinliche Funktion der verschiedenen Borstentypen.

\begin{tabular}{|c|c|c|c|c|}
\hline $\begin{array}{l}\text { No Types } \\
\text { de soies } \\
\text { No. des } \\
\text { Borstentyps }\end{array}$ & $\begin{array}{l}\text { No des } \\
\text { figures } \\
\text { No. der } \\
\text { Abbildung }\end{array}$ & $\begin{array}{l}\text { Description des soies } \\
\text { Beschreibung der Borsten }\end{array}$ & $\begin{array}{l}\text { Implantation } \\
\text { Implantation }\end{array}$ & $\begin{array}{l}\text { Fonction } \\
\text { Funktion }\end{array}$ \\
\hline 1 & $\begin{array}{l}\text { Fig. } 2 \text { ( } c \text { et } d \text { ) } \\
\text { Abb. } 2 \text { (c u. d) }\end{array}$ & $\begin{array}{l}\text { Simples longues }(0,25 \mathrm{~mm}) \\
\quad \text { rigides, pointues; à carènes } \\
\text { latérales } \\
\text { Einfach, lang }(0,25 \mathrm{~mm}) \text {, steif, } \\
\text { gespitzt, mit lateralen Rillen }\end{array}$ & $\begin{array}{l}\text { Basitarse, face interne. } \\
\text { Cf. : Fig. } 2(b) \\
\text { Basitarsus, Innenseite. S. } \\
\begin{array}{ll}\text { Abb. } 2, b & \end{array}\end{array}$ & $\begin{array}{l}\text { Nettoyage-bros- } \\
\text { pollen } \\
\text { Ausbürsten von } \\
\text { Pollen }\end{array}$ \\
\hline 2 & $\begin{array}{l}\text { Fig. } 3(a) \\
\text { Abb. } 3(a)\end{array}$ & $\begin{array}{l}\text { Petites }(0,03 \text { à } 0,04 \mathrm{~mm}) \\
\text { extrémité aplatie et élargie } \\
\text { en forme de spatule } \\
\text { Klein }(0,03-0,04 \mathrm{~mm}) \text {, Spitze } \\
\text { spatelförmig abgeplattet und } \\
\text { verbreitert }\end{array}$ & Femur, Innenseite & - \\
\hline 3 & $\begin{array}{l}\text { Fig. } 3(b) \\
\text { Abb. } 3(b)\end{array}$ & $\begin{array}{l}\text { Plumeuses, longues }(0,27 \mathrm{~mm}), \\
\text { noires, barbes }(0,06 \mathrm{~mm}), \\
\text { rigides et arquées } \\
\text { Gefiedert, lang }(0,27 \mathrm{~mm}), \\
\text { schwarz, Fiedern steif und } \\
\text { gebogen }\end{array}$ & $\begin{array}{l}\text { Tibia, face externe, adja- } \\
\text { cente à la plaque basi- } \\
\text { tibiale. Cf. : Fig. } 2(a) \\
\text { Tibia Aussenseite, angren- } \\
\text { zend a.d. Basitibialplatte }\end{array}$ & - \\
\hline 4 & $\begin{array}{l}\text { Fig. } 3(\mathrm{c}) \\
\text { Abb. } 3(\mathrm{c})\end{array}$ & $\begin{array}{l}\text { Plumeuses, longues }(0,27 \mathrm{~mm}), \\
\text { barbes longues et souples } \\
\text { Gefiedert, lang }(0,27 \mathrm{~mm}) \text { Fiedern } \\
\text { lang und zart }\end{array}$ & $\begin{array}{l}\text { Tibia et basitarse, face } \\
\text { externe } \\
\text { Tibia und Basitarsus, Aussen- } \\
\text { seite }\end{array}$ & $\begin{array}{l}\text { Stockage pol- } \\
\text { len } \\
\text { Stapeln von Pol- } \\
\text { len }\end{array}$ \\
\hline 5 & $\begin{array}{l}\text { Fig. } 3(d) \\
\text { Abb. } 3(d)\end{array}$ & $\begin{array}{l}\text { Plumeuses }(0,1 \text { à } 0,2 \mathrm{~mm}) \\
\text { barbes insérées en barbelé } \\
\text { vers l'extrémité distale } \\
\text { Gefiedert }(0,1-0,2 \mathrm{~mm}) \text { Fiedern } \\
\text { am distalen Ende }\end{array}$ & $\begin{array}{l}\text { Divers endroits, base des } \\
\text { éperons du tibia et arti- } \\
\text { culation tibia-basitarse } \\
\text { An versch. Stellen, Basis d. } \\
\text { Tibia u. Tibia-basitarsal- } \\
\text { Gelenk }\end{array}$ & \\
\hline \multirow[t]{2}{*}{6} & Fig. $4(a, b)$ & $\begin{array}{l}\text { Plumeuses }(0,2 \mathrm{~mm}) \text { arquées } \\
\text { à barbes hérissées vers l'ex- } \\
\text { térieur }\end{array}$ & $\begin{array}{l}\text { Articulation fémuro-ti- } \\
\text { biale. Cf. : Fig. } 4(c)\end{array}$ & $\begin{array}{r}\text { Protection des } \\
\text { articulations }\end{array}$ \\
\hline & Abb. $4(a, b)$ & $\begin{array}{l}\text { Gefiedert }(0,2 \mathrm{~mm}) \text { mit nach } \\
\text { aussen gebogenen Fiedern }\end{array}$ & $\begin{array}{l}\text { Femuro-Tibialgelenk, S. Abb. } \\
\quad 4 c\end{array}$ & $\begin{array}{c}\text { Schutz der Ge- } \\
\text { lenke }\end{array}$ \\
\hline
\end{tabular}

Six types des soies sont différenciés, dont quatre sont les constituants des scopae. Les de ix soies extra-scopales sont citées en raison de leur particularité morphologique ou/et fonctionnelle.

- Parmi les soies scopales nous avons reconnu la fonction de deux d'entre elles :

a) Les soies du type 1 (Fig. 2, c, d) recouvrent en abondance (Fig. 1 et Fig. 2 b) la face interne du basitarse. Cette situation et la morphologie de ces soies leur confèrent une fonction de brossage du pollen sur l'ensemble de l'abdomen. Il est à noter que des soies du même type, implantées sur les pattes antérieures et médianes, permettent le brossage du reste du corps.

b) Sur la face externe des tibia et basitarse (Fig. 1 et Fig. 2 a) sont implantées en forte densité des soies du type 4 (Fig. 3 c). L'enchevêtrement des longues barbes de ces 
soies permet une bonne fixation de la masse pollinique et forme ainsi l'appareil collecteur de pollen.

c) Nous n'avons pas reconnu la fonction des deux autres types ( 3 et 5 ) de soie. Le type 5 est communément réparti sur l'ensemble du corps de l'insecte (Fig. 3, d).

- Parmi les soies extra-scopales, celles qui appartiennent au type 2 (Fig. 3 a) ont une extrémité spatulaire. Elles sont implantées en densité moyenne sur la face interne du fémur. Les soies plumeuses de type 6 (Fig. $4 a, b, c$ ) garnissent l'extrémité distale du fémur. Elles auraient un rôle protecteur.

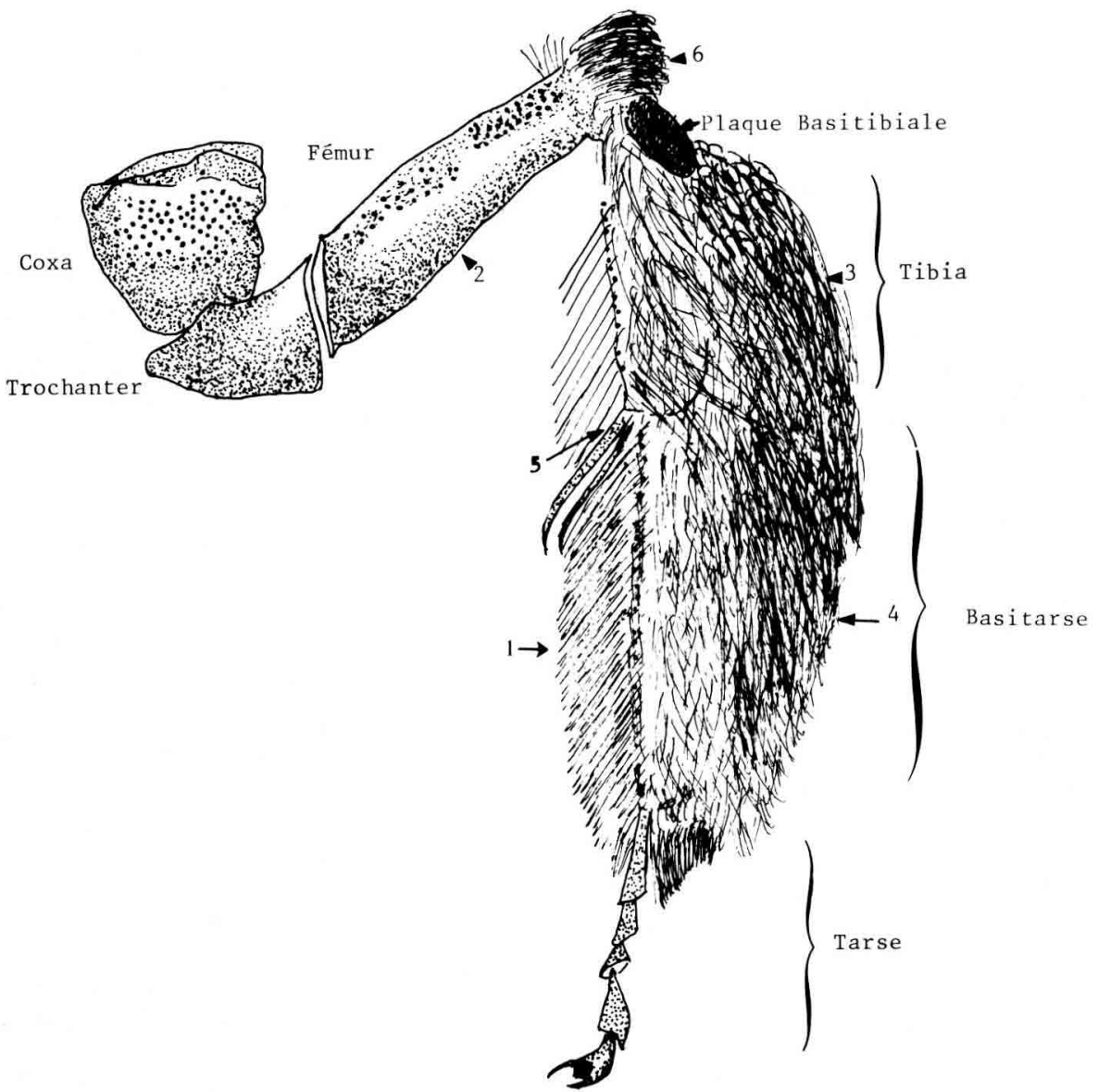

FIG. 1. - Schéma d'une patte postérieure d'Exomalopsis avec indication de l'emplacement des divers types de soies ( $\times 36,5)$.

Авв. 1. - Schema eines Hinterbeines von Exomalopsis mit Hinweis auf das Vorkommen der verschiedenen Borstentypen $(36,5 \times)$. 

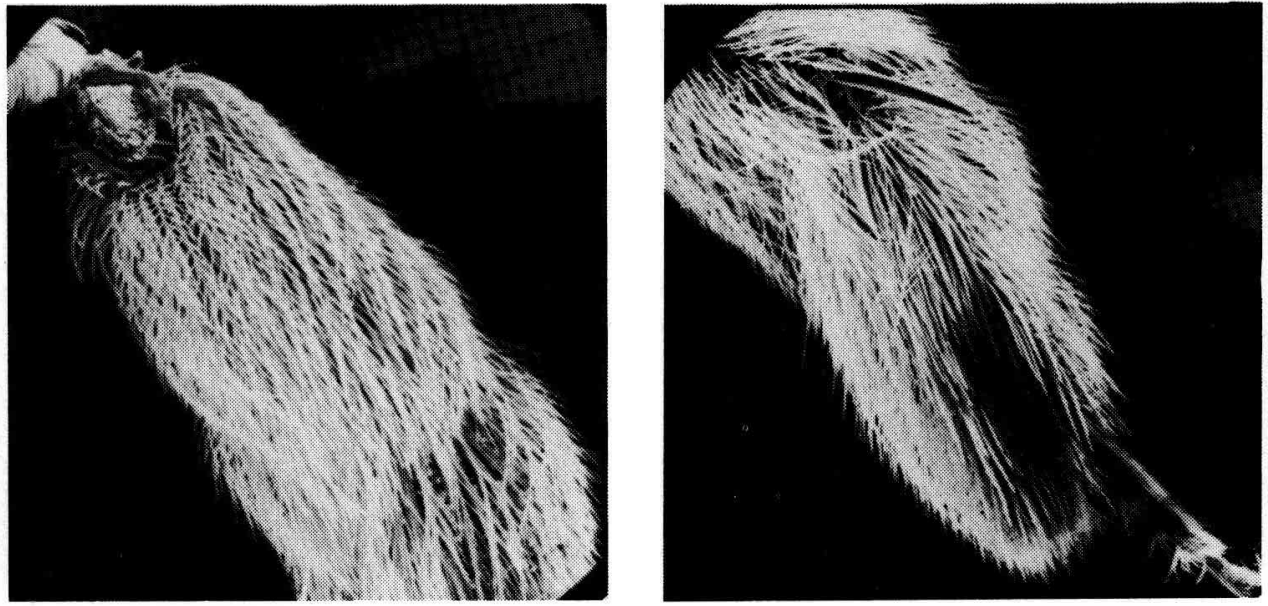

FIG. 2 a. - Vue partielle de la Scopa face externe,

de haut en bas : plaque basitibiale, tibia, partie de basitarse ( $\times 33)$.

Aвв. 2 a. - Teilansicht der Aussenseite der Scopa. Von oben nach unten : Basitibialplatte, Tibia, Teil des Basitarsus $(33 \times)$.

FIG. 2 b. - Vue partielle de la Scopa face interne,

de haut en bas : partie de tibia avec éperon visible, basitarse et autres tarses ( $\times 33)$.

Авв. 2 b. - Teilansicht der Innenseite der Scopa. Von oben nach unten :

Teil der Tibia mit sichtbarem Sporn, Basitarsus und andere Tarsen $(33 \times)$.
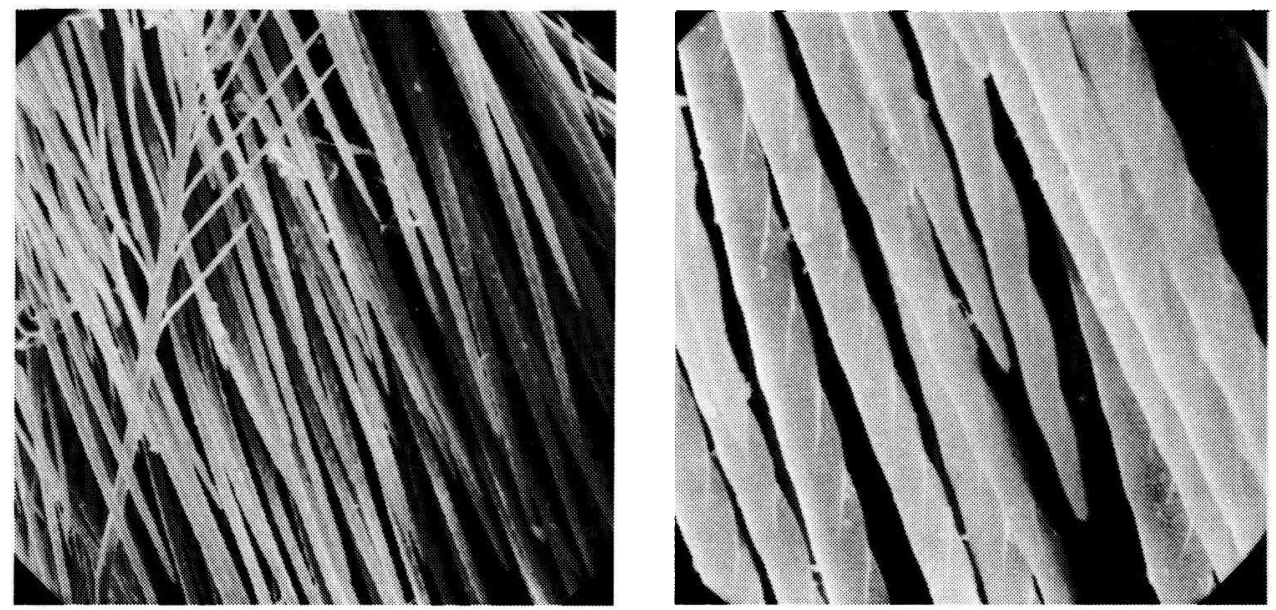

FIG. 2 c. - Soies simples de type $l(\times 400)$.

AвB. 2 c. - Einfache Borsten, Typ $1(400 \times)$.

Fig. $2 d$. - Soies simples de type 1 avec carènes latérales bien visibles $(\times 100)$.

AвB. 2 d. - Einfache Borsten von Typ 1, mit gut sichtbaren lateralen Kiele. 

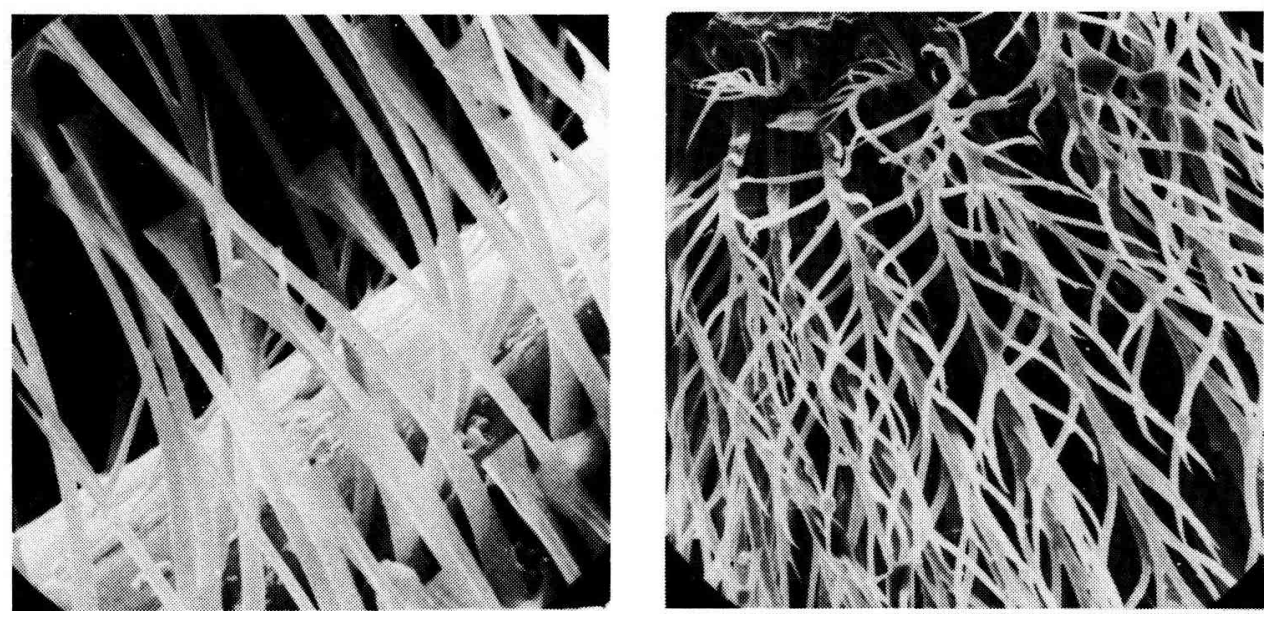

FIG. 3 a. - Soies simples spatulaires de type $2(\times 1000)$.

Aвв. 3 a. - Einfache Borsten von Spatelform, Typ 2 (1000 x).

FiG. 3 b. - Soies plumeuses de typo 3 à barbes arquées $(\times 400)$.

Aвв. 3 b. - Gefiederte Borsten, Typ 3, mit gebogenen Fiedern (400 $\times$ ).
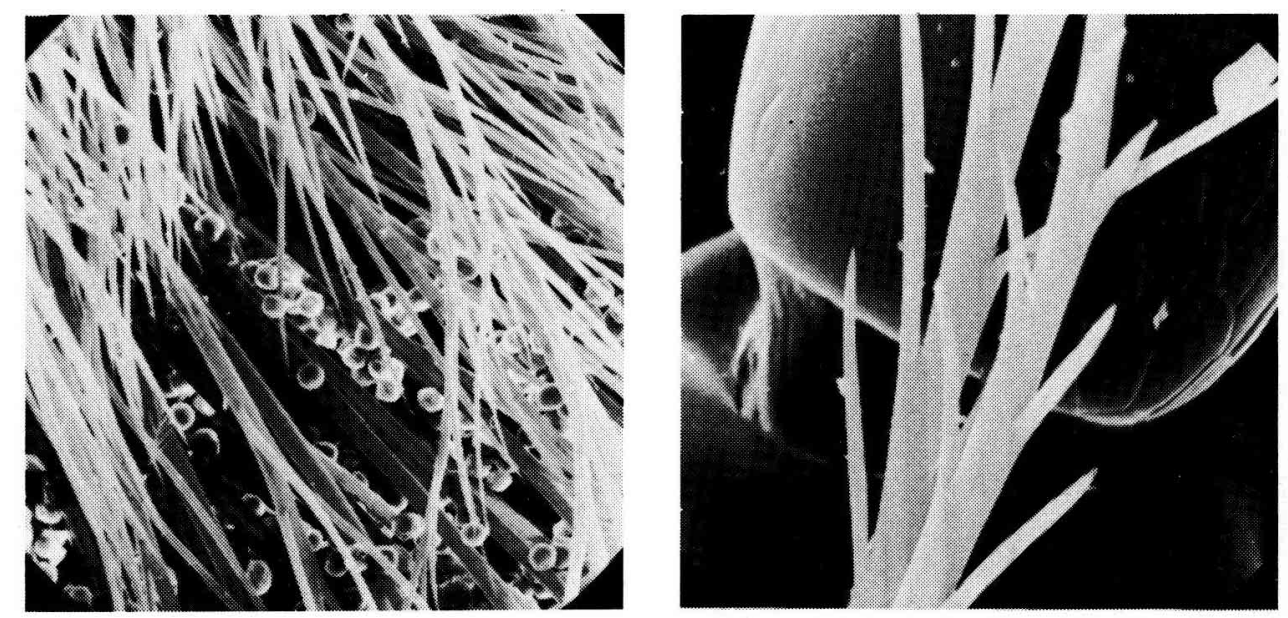

FIG. 3 c. - Soies plumeuses de type 4 à longues barbes simples $(\times 400)$. Noter la présence de pollen.

Aвв. 3 c. - Gefiederte Borsten, Typ 4, mit langen einfachen Fiedern $(400 \times)$. Beachte die Pollenkörner.

FIG. $3 d$. - Soies plumeuses de type 5, les barbes sont insérées sur les côtés et barbelées à l'extrémité $(\times 1000)$.

Авв. $3 d$. - Gefiederte Borsten vom Typ 5, die Fiedern sitzen an den Seiten und sie sind am Ende widerhakig (1 $000 \times)$. 

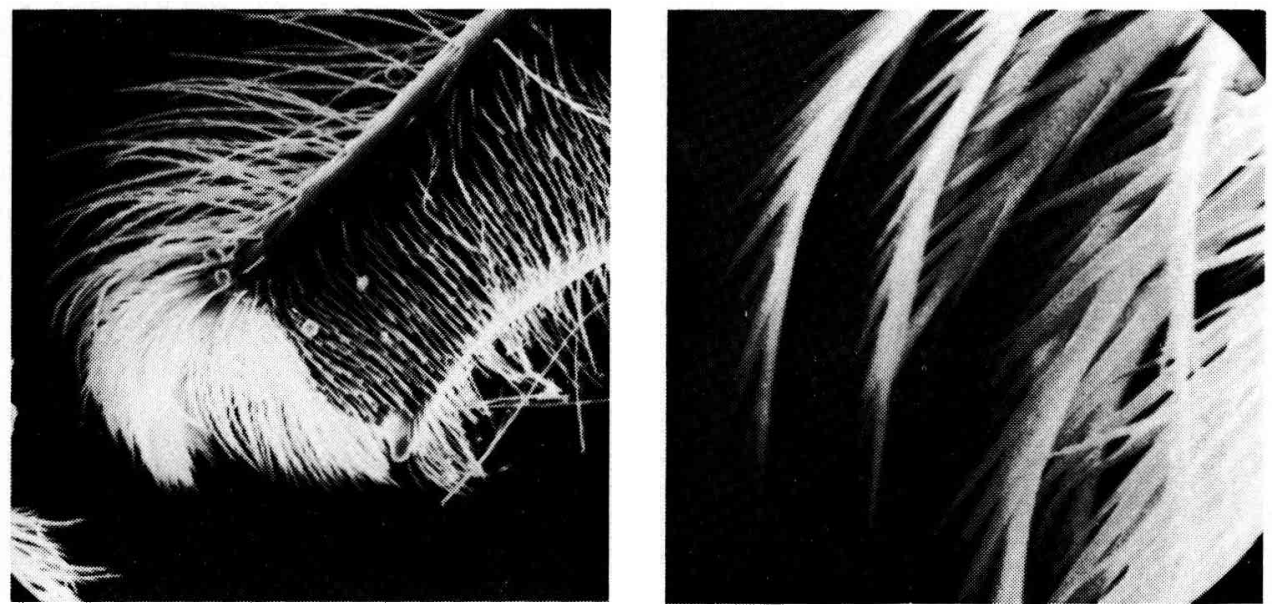

FIG. 4 a. - Partie distale du fémur, adjacente au tibia et présentant des soies spatulaires du type 2 couchées sur la face interne et de soies plumeuses et arquées de type 6 à l'extrémité $(\times 100)$.

ABв. 4 a. - Distaler Anteil des Femur, angrenzend an die Tibia, mit spatelförmigen Borsten vom Typ 2 an der Innenseite und gefiederten und gebogenen Borsten vom Typ 6 an der Spitze $(100 \times)$.

Fig. 4 b. - Détails des soies plumeuses et arquées de type $6(\times 1000)$. Aвв. 4 b. - Details von den gebogenen Fiederborsten von Typ $6(1000 \times)$.

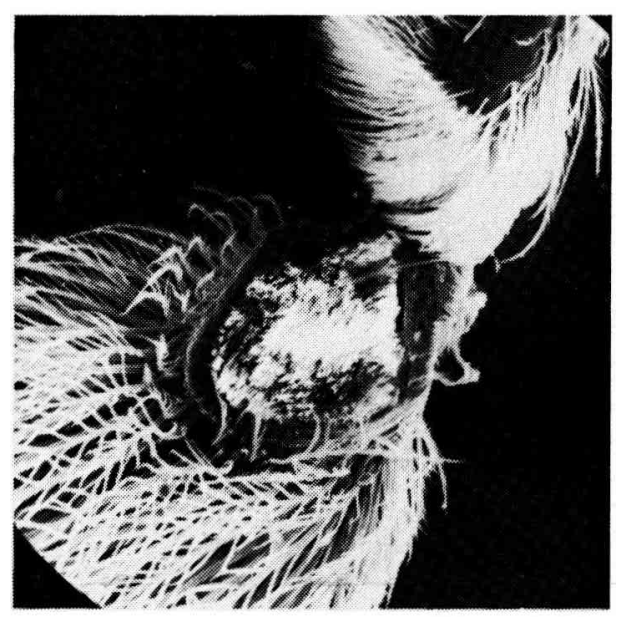

FIG. 4 c. - Soies de type 6 implantées à l'extrémité distale du fémur et offrant une couverture protectrice à l'articulation fémuro-tibiale. Extrémité basale du tibia et vue de la plaque basitibiale $(\times 100)$.

AвB. 4 c. - Borsten vom Typ 6 implantiert am distalen Ende des Femur, die den Femuro-Tibiagelenk Schutz verleihen.

Basales Ende der Tibia und Ansicht der Basitibialplatte $(100 \times)$. 


\section{DISCUSSION - CONCLUSION}

Parmi les insectes, les Apoïdes présentent les soies cuticulaires les plus abondantes et diverses. Cette adaptation morphologique est liée à leur activité floricole.

Exomalopsis biliottii visite les fleurs dont elle prélève le pollen et/ou le nectar pour sa propre nourriture et celle de son couvain. Pour recueillir et transporter ce pollen, la femelle possède sur les pattes postérieures une grande concentration de longues soies formant la scopa. Leur observation au microscope électronique à balayage permet de mettre en évidence la présence de soies dont la morphologie et l'implantation sont particulièrement adaptées à la récolte du pollen.

L'ensemble du corps des femelles et particulièrement le thorax est abondamment pourvu de soies plumeuses (type 5) participant à la récolte du pollen.

Les pattes antérieures et médianes sont fournies en longues soies simples du type 1 servant respectivement à brosser le pollen de la tête et du thorax, l'abdomen ètant pour l'essentiel dans le champ d'action des pattes postérieures.

La charge de pollen est portée uniquement par les scopae des basitarses et des tibias. Celles-ci sont entièrement recouvertes par la masse pollinique récoltée et à travers laquelle n'apparait plus que l'extrémité des longues soies plumeuses et simples de type 4 et 1 . Dès lors les pattes postérieures présentent un aspect renflé, en forme de pelote plus ou moins vivement colorée en blanc cassé ou jaune orange selon le pollen dominant.

La fonction de protection articulaire de certaines soies extra-scopales est soupçonnée chez d'autres abeilles par PAsteels et PAsteels (1972). Ces mêmes auteurs observent également la présence de soies spatulaires. Tout comme eux nous ne leur avons pas reconnu de fonction particulière.

La pilosité fortement plumeuse des scopae fait de cette tribu (Exomalopsini) le taxon le plus primitif de la sous-famille des Anthophorinae (STEPHEN et al. 1969).

Reçu pour publication en juin 1979. Eingegangen im Juni 1979.

\section{REMERCIEMENT}

Nous remercions M. Pointel de la Station de Zoologie I.N.R.A. du Centre de Versailles pour la qualité de son travail au microscope électronique à balayage JSM 35.

\section{ZUSAMMENFASSUNG}

Die Raster-Elektrononenmikroskopie hat die morphologische Beschreibung verschiedener Borstentypen von den Pollenbürsten (scopae) einer kleinen Biene aus der Gruppe der Anthophoriden 
ermöglicht, einer Biene, die auf Guadeloup die kultivierten Solanaceen bestäubt. In Zusammenhang mit einer Untersuchüng des Sammelverhaltens dieses Insekts wurden die Beziehungen zwischen der Morphologie, der Implantation und der Funktion der skopalen Borsten analysiert. Es hat den Anschein, dass diese Beziehungen für zwei Borstentypen ausgeprägt sind :

- Eine einfache Borste, implantiert an der Innenseite des Basitarsus, mit der Funktion, den Pollen vom Körper des Tieres oder von den Blüten abzubürsten.

- Gefiederte Borsten, in grosser Zahl an der Aussenseite der Tibia und des Basitarsus vorhanden, wo der Pollen während des Transportes konzentriert wird.

Eine extraskopale Borste erweckt wegen ihrer Morphologie Interesse, weil sie wahrscheinlich geeignet ist, das Gelenk zwischen Femur und Tibia zu schützen.

\section{BIBLIOGRAPHIE}

MiChener C. D., 1944. - Comparative external morphology phylogeny and a classification of the bees (Hymenoptera). Bull. Amer. Mus. Nat. Hist., 82, 157-326.

PAsteels J. J., et PAsteels J. M., 1972. - Les soies cuticulaires des Megachilidae (Hymenoptera, Apoidea) vues au microscope électronique à balayage. Acad. Royale Belgique. Classe des Sciences. Mémoires, $2^{\mathrm{e}}$ série, 18, 1-27.

Pasteels J. M. et PAsteels J. J., 1973. - Champs morphogénétiques mis en évidence par l'étude au microscope électronique à balayage des soies au niveau des pattes d'abeilles (Apoïdea, Megachilidae). Tissue \& Cell, 5, 63-82.

Pasteels J. J. et Pasteels J. M., 1974. - Étude au microscope électronique à balayage des Scopas abdominales chez de nombreuses espèces d'abeilles (Apoïdea, Megachilidae) Tissue \& Cell, 6 (1), 65-83.

Stephen W. P., Bohart G. E., Torchio P. F., 1969. - The biology and external morphology of bees. Agr. Exp. Stn., Oregon St Univ., Corvallis, 140 p.

\section{EMPFEHLUNGEN AN UNSERE AUTOREN}

Es wird gebeten, die Manuskripte in doppelter Ausfertigung (mit Durchschlag oder Fotokopie) einzusenden.

Das Manuskript soll einseitig in Maschinenschrift zweizeilig auf DIN-A-4-Bogen $(210 \times 297 \mathrm{~mm})$ geschrieben sein. Kursiv zu druckende Worte, z.b. Gattungs- und Artnamen, sind geradlinig zu unterstreichen, Autorennamen sollen mit grossen Buchstaben geschrieben werden. Handschriftliche Korrekturen und Einfügungen sind nicht zulässig. Die Seiten werden fortlaufend numeriert. Literaturverzeichnis, Tabellen und die Legenden zu den Abbildungen und graphischen Darstellungen werden auf besondere Bogen geschrieben, d. h. sie werden nicht mitnumeriert.

Am Anfang jeder Arbeit steht ihr Titel, der keine Abkürzungen oder chemische Formeln enthalten soll, ausgenommen Isotope. Es folgen der Name des Verfassers mit ausgeschriebenem Vornamen und gegebenenfalls der Name des technischen Mitarbeiters, dem folgender Wortlaut voransteht: Unter technischer Mitarbeit von... und der Name des Institutes, an dem die Arbeit durchgeführt wurde. Die vollständige Anschrift des Instituts kann am Ende der Arbeit oder als Fussnote auf der ersten Seite angegeben werden.

Die Arbeiten sollten nach Möglichkeit wie folgt gegliedert sein :

a. Kurze Inhaltsangabe (200-250 Worte), entsprechend den Empfehlungen der U.N.E.S.C.O. (Anleitung für die Abfassung wissenschaftlicher, zur Veröffentlichung bestimmter Arbeiten). Diese, in der Sprache des Autors abgefasste kurze Inhaltsangabe wird ins Englische übersetzt (abstract). 
Ђ. Einleitung; in ihr wird das Thema der durchgeführten Untersuchungen in Beziehung zu bereits veröffentlichten Arbeiten auf diesem Gebiet umrissen.

c. Methodik; sie beschreibt die Versuche sowie das benutzte Material, wobei in kurzer Form alle notwendigen Einzelheiten angegeben werden, die es dem Leser ermöglichen, die Versuche zu wiederholen.

d. Ergebnisse; sie sollten möglichst kurz gefasst sein.

e. Diskussion und Schlussfolgerung.

f. Ausführliche Zusammenfassung; sie wird in der Originalsprache abgefasst und übersetzt und zwar jeweils entweder in deutsch oder französisch oder auch in beide Sprachen, falls es sich um einen englischen Originalbeitrag handelt. Die Zusammenfassung - eigentlich eine ausführliche Analyse der Arbeit - soll ausreichend über Methodik, Ergebnisse, Diskussion und Schlussfolgerungen unterrichten, so dass derjenige Leser, der die Originalsprache des Beitrages nicht beherrscht, diesen doch verstehen und mit Gewinn lesen kann.

g. Englische Übersetzung (abstract) der oben erwähnten kurzen Inhaltsangabe.

h. Dank für Mitarbeit, Unterstützung usw.

i. Literaturverzeichnis.

Diese Empfehlungen gelten auch für englische Originalbeiträge. Veröffentlichungen wie z. B. kurze Mitteilungen, Übersichtsreferate, Berichte u.s.w. werden mit logischem Aufbau und passendem Titel verfasst.

Literaturangaben im Text sollen wie folgt gemacht werden : (DuPONT und Duval, 1959). Handelt es sich um mehr als drei Verfasser, wird nur der erste angeführt, die anderen durch et al. angegeben. Das am Ende der Arbeit stehende Literaturverzeichnis wird alphabetisch geordnet, mehrere Arbeiten desselben Autors erscheinen in zeitlicher Reihenfolge.

Die Autoren werden gebeten, die das Literaturverzeichnis betreffenden Einzelheiten den ausführlichen Empfehlungen an unsere Autoren zu entnehmen.

Es wird gebeten, Tabellen, Zeichnungen, graphische Darstellungen, Karten und Fotos gesondert beizulegen. Tabellen und Abbildungen erhalten jeweils eine eigene Numerierung in arabischen Ziffern.

Die Tabellen können Hoch - oder Breitformat haben. In der Breite beträgt der verfügbare Raum für Zeichen und Zwischenräume 100, in der Höhe 155. Tabellen in Hochformat, also solche, die 155 Zeichen enthalten können, sind nur dann zulässig, wenn sie in der Breite mehr als acht Zahlenreihen umfassen, so dass nicht eine ganze Seite der Zeitschrift von einer sehr hohen, schmalen Tabelle beansprucht wird. Wiederholungen zwischen Tabellen und graphischen Darstellungen sind zu vermeiden.

Legenden und Erläuterungen zu graphischen Darstellungen und Zeichnungen sollen auf besonderen Bogen in Maschinenschrift geschrieben sein und die Nummern der betreffenden Abbildungen tragen.

Alle Abbildungen sollen in ihrer endgültigen Form, also druckfertig geliefert werden. Drei verschiedene Verkleinerungs- Ma $\beta$ stäbe sind zulässig : 0,5 fach, 0,33 fach und 0,25 fach.

Es wird daran erinnert, dass die Seite der Zeitschrift $19 \mathrm{~cm} \times 12,5 \mathrm{~cm}$ gross ist, und dass dieses Mass nicht überschritten werden kann.

Bei den Fotos werden kontrastreiche Bilder auf weissem Papier, vorzugsweise in der Grösse $9 \times 12$ $\mathrm{cm}$ empfohlen. Sollen Fotos zu einer Platte zusammengestellt werden, werden folgende Masse empfohlen : 2 Fotos $9 \times 12$ und 4 Fotos 5,5 ×5,5. In diesem Fall soll jedes Foto eine Nummer tragen (arabische Ziffer), die auf dem Klischee zu sehen ist.

Die Legenden zu den Fotos sollen ebenfalls in Maschinenschrift auf besonderem Bogen geschrieben sein und die Nummern der Bilder tragen.

Bezüglich der Fahnenkorrektur werden die Autoren darauf aufmerksam gemacht, dass sie nur die erste Korrekturfahne zugeschickt bekommen.

Für die Fahnenkorrektur wird empfohlen, sich nach den Korrekturvorschriften zu richten, wie sie in "Der grosse DudEn ", Rechtschreibung, in den Vorbemerkungen abgedruckt sind.

Autorkorrekturen sind moglichst zu vermeiden. Grössere Änderungen müssen den Autoren in Rechnung gestellt werden. schicken.

Es wird gebeten, die Manuskripte an Herrn Prof. Dr. O. Wahl, Buschweg 13, 8165 Fischbachau 2 zu

Le Directeur de la publication : J. LOUvEAUX

Imprimerie de Montligeon (Orne) Dépôt légal n 10560

CCPAP. AD EP O 117 